\title{
MANER: Managed Data Dissemination Scheme for LoRa IoT Enabled Wildlife Monitoring System (WMS)
}

\author{
Eyuel D. Ayele, Nirvana Meratnia, Paul J.M. Havinga \\ Pervasive Systems Research Group \\ University of Twente, Enschede, The Netherlands \\ Email: \{e.d.ayele,n.meratnia,p.j.m.havinga\}@utwente.nl
}

\begin{abstract}
In this paper, we introduce dual radio based IoT network architecture for wildlife monitoring system (WMS). This solution will facilitate an IoT devices to be deployed for sustainable wildlife monitoring applications. In addition we present MANER, a managed data dissemination scheme for WMS. In MANER, data forwarding is optimized with a replication function to control and prioritize data dissemination. In WMS scenario wild animals show a sparsely con-specific mobility, which often results in a sporadic wireless link among nodes. Unlike existing opportunistic algorithms, MANER optimally makes forwarding decisions by leveraging locally available information. Hence, the proposed algorithm adopts to dynamic network topology due to the inherent intermittent connectivity among mobile herd of animals. We evaluated the performance of MANER by considering standard and real-life mobility models. Experimental results indicated that MANER decreases the average latency by up-to $65 \%$, when compared to benchmark opportunistic algorithms. In addition MANER readily increased the network delivery ratio for various data traffic rates.
\end{abstract} $B L E$

Keywords-Opportunistic Mobile Networks, OMN, IoT, LoRa,

\section{INTRODUCTION}

Wildlife monitoring system (WMS) is one of the IoT opportunistic mobile network (OMN) applications, where a number of heterogeneous sensors are deployed to monitor the activities of wild animals dwelling in a remote and geographically large habitat [1]. It is known that wild animals depict similar physiological activities as a compact herds of con-species [1,2]. Moreover, animals often show a level of movement (e.g. grazing, pursuing a prey) or running from danger such as illegal hunters or poachers. Thus, sensors (e.g. accelerometer, gyroscope, etc) could be deployed as collars to monitor these activities [3-5]. Peer-to-peer communication among sensor nodes in WMS deployment will facilitate local pre-processing and data sharing for collaborative decision making. To these end, WMS design should aim to achieve high reliability, low latency, and high energy efficiency. Based on the behavior of wild animals and the target application requirements, numerous data gathering protocols could be potential candidates for WMS data dissemination.

Conventional routing protocols, (e.g DSR or AODV [6]), are not suitable for WMS application where wild animals depict sparse and con-specific movement, resulting in sporadic and unstable end-to-end connectivity among nodes. Even-though mobility is considered as the fundamental facilitator for information dissemination in opportunistic networks, recent works have revealed that current opportunistic protocols perform less than expected for a sparsely mobile networks with nondeterministic movement [7]. For instance, Epidemic [7], and PRoPHET [8], offer a high data delivery ratio at the expense of high network overhead and latency. Spray and Wait (SnW) on the other hand results in low latency but high network overhead [7, 8]. Currently, several OMN based wildlife monitoring projects are proposed for sensor data gathering, e.g. ZebraNet [3, 9], Rat Watch [10]. These works, implement opportunistic networks by leveraging a history based flooding, which are prone to low delivery ratio and high latency [7].

Motivated by a such application scenarios and to fulfill the overall WMS design requirements, often require a metaheuristic approach, where each node makes a data forwarding decision locally. Therefore, in this work, we propose MANER - managed data dissemination scheme for WMS. The main contribution of these work is to further improve existing opportunistic algorithms by managing the data replication decision by leveraging locally available routing parameters. Moreover, in this paper, we propose an IoT network architecture for wild animal monitoring applications. The features that makes MANER suitable for WMS scenario are: (i) there is no network topology limitation imposed, (ii) node mobility is easily supported, and (iii) intermediate nodes utilize a simple store-carry-and-forward (SCF) scheme for data dissemination with out relying on routing tables. MANER minimizes data latency while avoiding deterioration in data delivery ratio. Through simulation, the performance of the proposed protocol is compared and evaluated with the state-of-the-art data dissemination protocols in terms of delivery ratio and average latency.

The rest of the paper is organized as follows: related works are introduced in Section II. Section III and IV presents the proposed WMS network architecture and MANER system design. Section $\mathrm{V}$ further discusses the implementation and evaluation results. Finally, Section VI outlines the concluding remarks and future research challenges. 


\section{RELATED WORK}

In this section we present data dissemination protocols as presented in the related literatures. In conventional routing algorithms (e.g DSR or AODV [6]), deciding where to send a data is often a straight forward routine; i.e. the data is forwarded to the next neighbor along the path to sink usually with shortest routing cost. Moreover, the gathered data is transfered to a sink using intermediate nodes as fixed relays. However, traditional routing protocols will perform poorly in WMS scenarios where the network is sporadically connected due to animal mobility pattern [9]. Currently, several research works have shown an improved data collection based on opportunistic sensing with fixed network infrastructure support. However, unless standard mobility model is considered, they often result in low delivery ratio and high latency $[5,9,10]$. Thus, an efficient data dissemination protocol is needed for a newly surfacing applications as in WMS IoT network architecture with random mobile nodes.

The work proposed in this paper is motivated by the novel protocols that incorporate common types of store-carryforward (SCF) dissemination schemes. A striking similarity among these protocols is that, they are based on data replication technique, however, they differ in their approach to optimize and restrict the degree of replication. For instance, Direct Delivery (DD) algorithm enables a node to directly exchange a data to the destination in range [7]. After the communication is finished the sender node erases the replicated message to avoid local buffer queue overflow. Hence, the direct delivery scheme is based on single replication routine which often results in low delivery ratio. By contrast, Epidemic spreads data through out the network similar to microorganism infection [7, 8]. When end-devices are in contact they exclusively replicate multiple copies of data to the near by receiver nodes. These processes will be repeated through the network when nodes are in their range of communication, ultimately data will reach the intended sink node. These epidemic approach results in a high data reliability, however, it will drastically deplete sensor node resources, e.g. energy and data storage space. Similarly, ProPHET [8] is introduced to estimate data delivery probability for every contact with a destined node before sending a data. To alleviate these issues associated with DD and Epidemic, Spyropoulos et. al introduced the Spray and Wait (SnW) protocol [11], by limiting and optimizing the number of data replication for every data. Notice that DD, SnW, and Epidemic are a family of stochastic dissemination that delivers data by spreading throughout the network by utilizing link contacts. These is cases the data will reach the intended destination slowly.

In this paper, the ONE simulator [12] is utilized since it includes opportunistic protocols. In addition it is ease to import a real-life mobility trace files into simulator to make evaluation more realistic. It is ease to set-up the simulation with various parameters e.g. mobility speed, data sizes etc.

\section{WMS NETWORK ARCHITECTURE}

This section introduces the proposed network architecture and protocol stack for WMS. Most conventional WMSs are

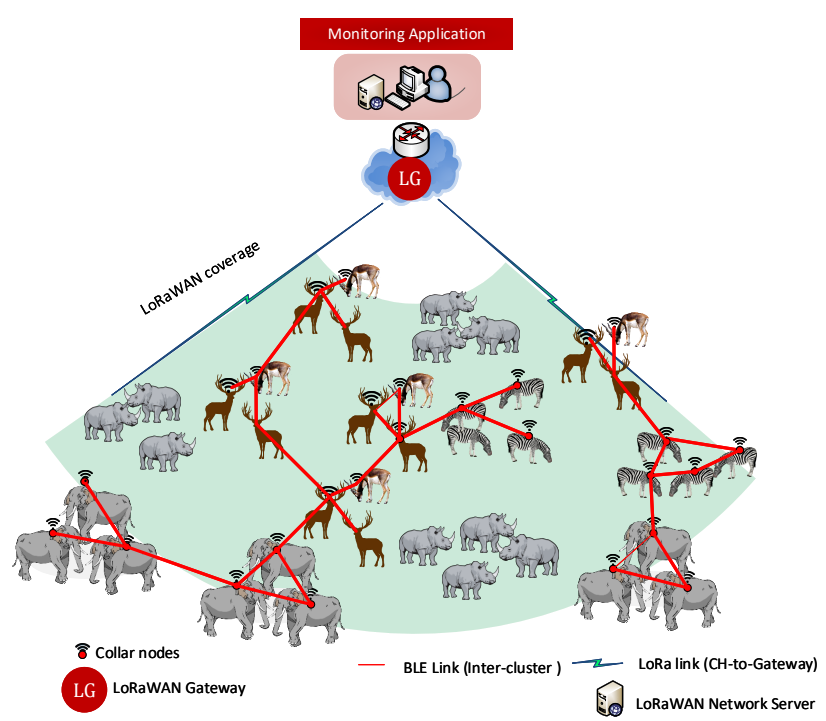

Fig. 1: Dual radio based opportunistic IoT network architecture for animal monitoring applications.

based on wireless sensor networks and consider nodes to be fully connected $[9,10]$. However, wild animals are inherently mobile and socialize in a distributed con-specific herds, which results in an intermittent connectivity among nodes and high level of uncertainty in the communication link. Thus, we propose an improved opportunistic IoT network architecture with LPWAN infrastructure (Fig. 1). In this work wild animals are assumed to be collared with inbuilt sensors and a dual radio platform (i.e. BLE and LoRa). The end-devices use short range BLE radio for peer-to-peer opportunistic mesh communication and long range LoRa radio to link to LoRa IoT gateways. The cooperation among the sensor networks allows a run-time monitoring of events in the area while reducing false alarms. Gateway backbone network is based on LoRa gateway (LG) [13], which is particularly suitable due to its long range communication provision at much lower power compared to other existing IoT protocols [13]. LoRa gateway (LG) serves as the main component to relaying data to LoRa central server. LoRa network server runs the real-time event monitoring application.

In WMS protocol stack, in the upper part an application layer and MANER, as the network manager, is placed (Fig. 2). When source nodes want to send data to sink node, they utilize MANER, a simple opportunistic store-carry-forward (SCF) protocol, then the sink node concatenates and merges incoming data before relaying them to LoRa gateway. Instead of direct LoRa connectivity as in star topology, applying data concatenation at the sink node will drastically reduce the overall energy overhead. The concatenation algorithm is out of the scope of this paper, however, we plan to detail the procedure in future work. It is worth while to notice that there are no topology restrictions in utilizing this network 


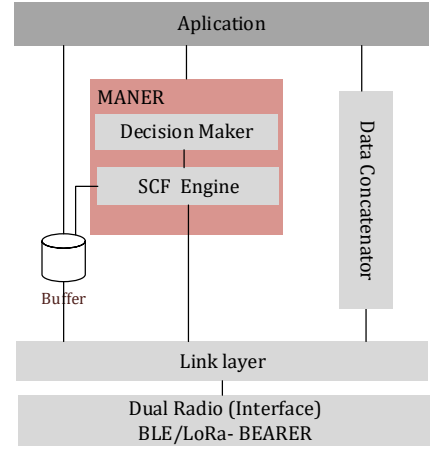

Fig. 2: Overview of protocol stack for WMS applications.

architecture. Below the network manger there are two bearer stacks, one for each radio technology, i.e. BLE and LoRa as the MAC/PHY layers. For details on LoRa and BLE technology, the readers are referred to [13-15]; in this paper we mainly focus on its utilization to WMS. Neighbor discovery is one of the key mechanisms of PHY/MAC to efficiently administer self-organizable mobile networks. Unlike other short-range wireless technologies, e.g. WiFi direct, the recently released specification for BLE - Mesh v1.0 offers low-power and simple device discovery which easily enables opportunistic network deployment in WMS [16]. The proposed routing protocol can work on top of any MAC/PHY layer, however, in this paper we focus on MANER - a meta-heuristic data dissemination protocol for BLE opportunistic network, and abstracts the MAC/PHY bearer layer as indicated in Section V.

\section{MANER PROTOCOL}

In this section, we present design overview of MANER followed by a discussion on its operation.

\section{A. MANER In Action}

The objective of proposed protocol is to provide a simple managed flooding scheme based on BLE mesh to accommodate herd mobility and perform better than existing algorithms. As shown in Figure 3.b, when a source node $S$ wants to send a data to destination node $D, S$ passes data to its neighboring node and process is repeated until data reaches $D$. We assume that nodes communicate only when sensor collars are within the transmission range of one another (Fig. 3.a and 3.b). If there is a disconnection of the link en route to $D$, it is likely that one or more node (s) come into contact with another node at a later time due to inherent animal movement. Thus data is spread throughout the entire network and will eventually be received by $D$. MANER enables nodeto-node $(n: n)$ or many-to-many $(m: m)$ communication in WMS. Hence, there is no network topology limitations, while deploying MANER in wild-life monitoring scenario. It can work on top of any data packet bearer layer, e.g. BLE radios. Unlike conventional protocols, it does not require complex routing table management and it is inherently multi-path, while

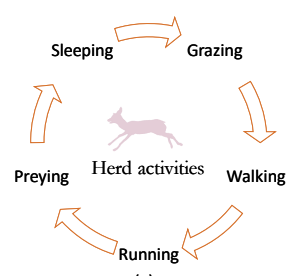

(a)

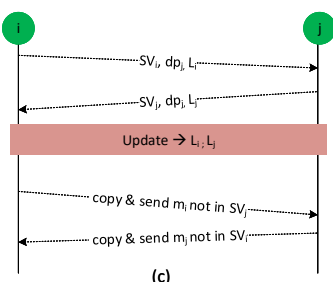

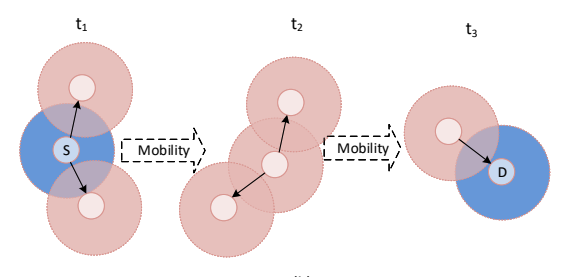

(b)
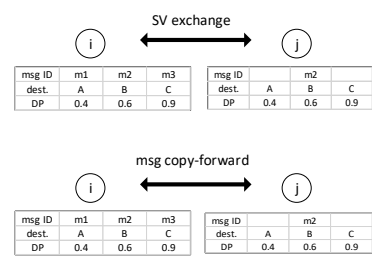

(d)
Fig. 3: (a) Example of animal physiological activities, (b) MANER protocol operation, $(c) \&(d)$ Summary Vector $(S V)$ and inter-contact time $(L)$ exchange between node $i$ and $j$ upon contact.

allowing neighboring nodes to communicate directly with one another to enable network level services such as proximity detection and peer-to-peer collaboration among nodes.

\section{B. Replication Algorithm}

We adopt a simple store-carry-forward (SCF) algorithm involving the exchange of summary vectors (SVs) - a list of data IDs contained by a node in its buffer, and delivery probability $(d p)$, similar to most opportunistic protocols [7] (Fig. 3.d). When a node receives an SV from another node, it learns the data that should not be replicated. The main contribution of these work is that, MANER further improves existing opportunistic algorithms by managing data replication decisions based on routing parameters such as: inter-contact time $(L)$, hop-count $\left(H c_{i, m}\right)$, number of replication $\left(n^{r}\right)$, and delivery probability $(d p)$.

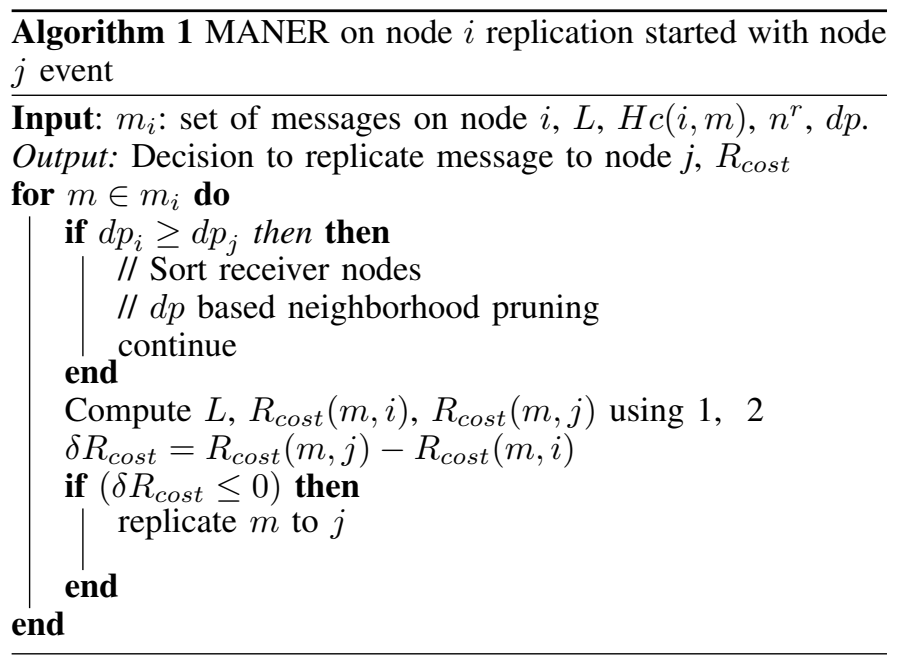


Algorithm 1 summarizes the action of MANER carried out by any node, when an active forwarding event with another receiver is invoked. Consider that a forwarder node $i$ is contemplating the replication of a data $m$ to node $j$. The forwarding decision will depends on per-link replication cost $\left(R_{\text {cost }}\right)$ computed based on $L_{i}$ and $H c_{i, m}$ (Alg. 1). Let $L_{i, j}$ be the time instant when the previous contact of node $i$ with node $j$ is terminated. Then, at the next contact event with $j$ at time instant $(t)$, the exponential smoothed estimation of the next inter-contact time $\left(L_{i, j_{(t)}}\right)$ value of node $i$ is updated as $L_{i, j_{(t)}}=\alpha \times L_{0, i}+(1-\alpha) \times L_{i, j_{(t-1)}}$, where $L_{0, i}$ is the value at a time period $(\mathrm{t})$, and $0 \leq \alpha \leq 1$ is the smoothing factor of $L_{i, j}$. Hence, the per-link replication cost $\left(R_{\text {cost }}\right)$ of having a data $m$ at node $i$ is expressed as [17] :

$$
R_{c}(m, i)=L_{i, j_{(t)}} \times\left[1+H c_{i, m}\right]
$$

The hop-count $\left(H c_{i, m}\right)$ is incremented by unity in order to prevent the cost function from becoming zero at the source of data. It may be observed that, if $m$ is replicated to $j$, the hop-count of $m$ at $j$ increases by unity. In other words, the replication action will allow data to spread one hop, which increased the value of its routing cost. This is in line with other opportunistic routing algorithms [11]. Therefore, the replication function at receiver $j$ is computed as:

$$
R_{c}(m, j)=L_{j, i_{(t)}} \times\left[2+H c_{i, m}\right]
$$

The steady state difference in the two cost functions is $\delta R_{c}=R_{c}(m, j)-R_{c}(m, i)$. Thus forwarder node $i$ decides to replicate, if the $\delta R_{\text {cost }} \leq 0$. In other words, such a replication would help the data to traverse from a higher cost state to a lower cost state. Algorithm 1 uses the local routing cost functions in Eq. 1 and 2 to initiate replication decision to the next hop.

Optimization: In addition, the forwarder node optimizes data replication decision by restricting the number of times $\left(n^{r}\right)$ a data is replicated and pruning the receiving nodes according to $(d p)$. To prevent the stored SV list for each data from becoming too long to overflow the buffer, number of replicated data is optimized by limiting $n^{r}$ value. The $n^{r}$ is decreased down to 1 after each successful forwarding to optimize the delivery ratio of data. All data originating from the same source have the same initial value of $n^{r}$. As shown in Algorithm 1, when a forwarder node $i$ encounters one or more nodes, the forwarder will collect and sort all active connections in descending order of the $d p$. When there are several nodes in communication range, a node will be selected as a forwarder node and remaining nodes as receiver nodes by applying some existing neighbor filtering mechanism, such as k-means clustering [11]. The receiver node, of which the $d p$ is higher than that of others, will have higher priority to receive data from the forwarder. These requested messages are sorted by a message sorting method, such as, First In First Out ( $F I F O$ ), before being pushed into the outgoing buffer. Due to the limited space, we will discuss the sorting schemes in more detail in journal.

Notice that MANER mimics as Epidemic routing by flooding the entire network with data copies, when $n^{r}$ is set to $+\infty$ for all nodes. When $n^{r}$ is finite value, a node will spread packets either like $\mathrm{SnW}\left(-\infty \leq n^{r}{ }_{i} \leq 1\right)$ or $\mathrm{DD}\left(n^{r}{ }_{i}=1\right)$ depending on the value set [11]. Thus, for a sparsely mobile network as in WMS application scenario, where herds of animals will often move randomly in con-specifics manner, $n^{r}$ is set in the range $\left(1 \leq n^{r}{ }_{i} \leq \infty\right)$ depending on the expected optimal latency. However, one of the easiest approach to determine the optimal $n^{r}$ value for our WMS mobility use case is to solve the optimization equation as presented in [11], where the expected delay is related to optimal latency as $E D_{s w}=a \times E D_{o p t}, a$ is a factor for delay constraint set by an application. As per [11], $a=5$, implying the average delay is up-to 5 times of the optimal latency.

$$
n^{r} \approx \frac{n}{a \times k_{n}}
$$

Assuming all nodes are mobile, this results as in Eq. 3, where $n$ is number of mobile nodes, which is consistent with the analysis done in [11]. In the future, we are going to investigate an alternative multi-parameter optimization approach in an extended journal since it takes a lot of space to describe the solution.

\section{Evaluation}

In this section, we discuss the simulation and experimental setup used to evaluate the performance of MANER in WMS applications.

\section{A. Simulation Set-up}

The evaluation set-up is similar to the one used in Ayele et al. [18] with a slight difference in parameter configurations. In addition we consider two mobility models, the first is random way point (RWP) movement in which a mobile node moves in random trajectory and at node speed range of $10-30 \mathrm{~km} / \mathrm{h}$ with max-pause $=5 \mathrm{~s}$. Naturally, animals do not just wander around randomly, they prefer to go somewhere for a purpose, often using the fastest path possible [3]. These destinations are very diverse, ranging from points of interest (e.g., water, grazing, etc.) to more distressing incidents (e.g. preying, running, etc.). Thus, we use a second movement model from the ZebraNet GPS data to model the behavior of real animal mobility [9]. We evaluated the performance of proposed approach in the ONE simulator with the movement models imported [12]. The network is set-up as shown Figure 1. In both mobility models we simulate animals moving in a defined trajectory in a grid area of $10000 \mathrm{mx} 10000 \mathrm{~m}$. The MAC/PHY layers are abstracted to transmission range and data rate in the simulator. Since our proposed architecture considers BLE interface for shortrange communication, all mobile nodes have BLE at $250 \mathrm{kbps}$ data rate with $200 \mathrm{~m}$ radio range. Depending on various animal species' empirical and modeled data, it is known that the optimal average group or herd size is in the range of $n \leq 400$ ], for instance, impala and zebra has a mean herd density of $\leq 70 / \mathrm{km}^{2}$ [19]. Therefore, we set the data replication number to $n^{r}=13$ minimizing the trade-off between latency and delivery ratio in our scenario, which is in agreement with $n^{r} \approx 12.5$ (for $a=5$ and $n=400$ mobile nodes in 


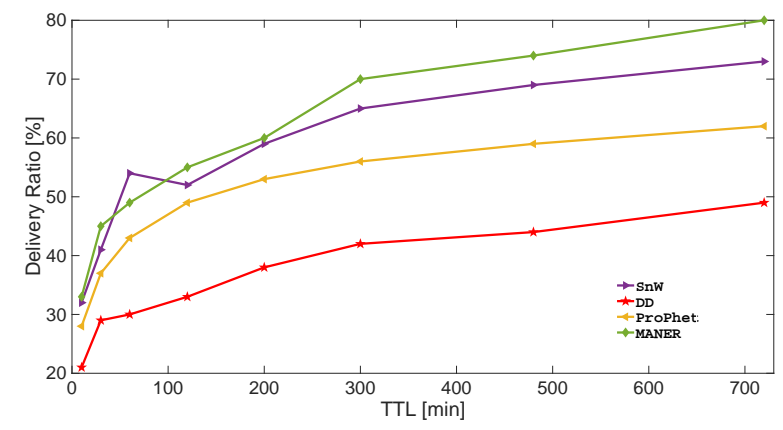

(a) RWP Delivery Ratio for High $T^{+}{ }_{m g}$

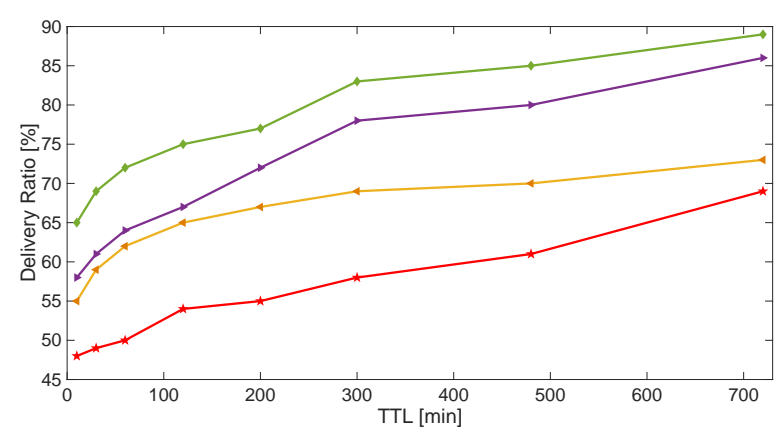

(c) ZebraNet Delivery Ratio for High $T^{+}{ }_{m g}$

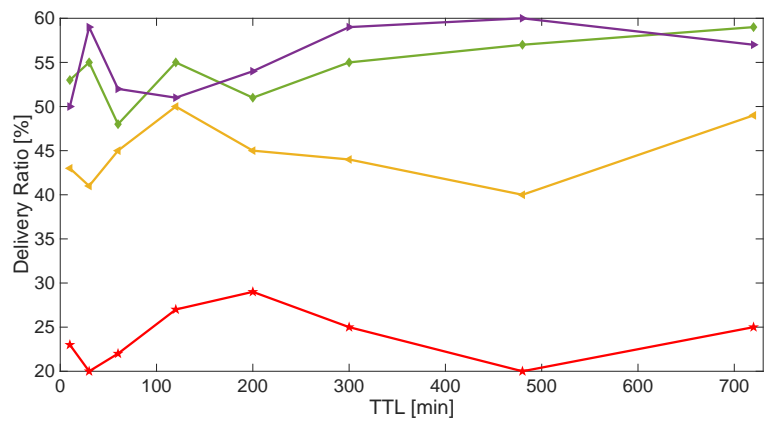

(b) RWP Delivery Ratio for Low $T^{+}{ }_{m g}$

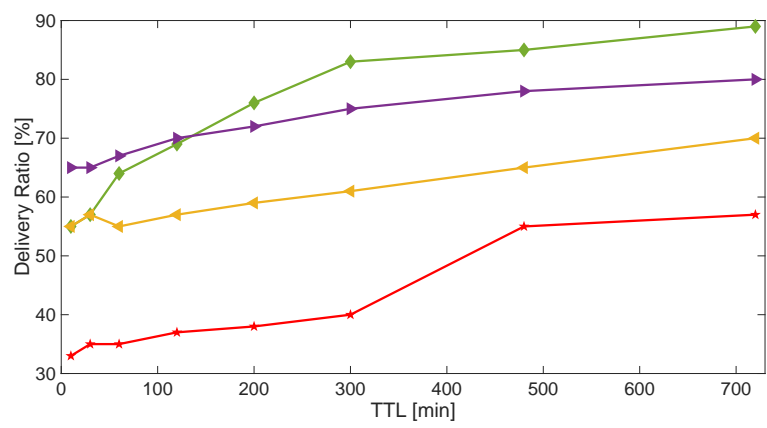

(d) ZebraNet Delivery Ratio for Low $T^{+}{ }_{m g}$

Fig. 4: Delivery Ratio for RWP and ZebraNet mobility models

Eq. 3) [11]. The network is set-up to perform in a varying message generation rate $\left(T^{+}{ }_{m g}(s e c).\right)$.

TABLE I: Generic Input Simulation Parameters

\begin{tabular}{|ll|}
\hline Grid area & $10000 \times 10000 \mathrm{~m}^{2}$ \\
\hline Mobility model & RWP and ZebraNet \\
\hline Mobility speed & RWP $[10,30 \mathrm{Km} / \mathrm{h}]$ \\
\hline Simulation duration & $14 \mathrm{hr}$ \\
\hline RWP pause time & 5 Seconds \\
\hline Data rate (DR) & BLE $(250 \mathrm{kbps})$ \\
\hline Message TTL (min) & {$[0,30,60,120,300,480,700]$} \\
\hline$T^{+}{ }_{m g}($ sec. $)$ & $\left(25 \leq T_{m g} \leq 45\right)$ for High, \\
& $\left(400 \leq T_{m g} \leq 1500\right)$ for Low \\
\hline Tx range & BLE $(200 \mathrm{~m})$, LoRa $(6000 \mathrm{~m})$ \\
\hline Number of nodes & {$[1,400]$} \\
\hline Packet (PL) & 64 bytes \\
\hline$n^{r}$ & 13 \\
\hline Buffer size & $24 \mathrm{~kb}$ \\
\hline Smoothing constant $(\alpha)$ & 0.8 \\
\hline
\end{tabular}

The source nodes randomly generate data with 64 bytes payload at $\left(25 \leq T^{+}{ }_{m g} \leq 45\right)$ for high, and $(400 \leq$ $\left.T^{+}{ }_{m g} \leq 1500\right)$ for low data traffic settings. We remark that 64 bytes suffice for recording animal activity features (e.g. type and duration of the activity), such as running, grazing, or walking. In this work we only consider data to be generated from all source nodes towards one sink node initially located at the center of grid area, however, through time all nodes move based on the mobility trajectory model supplied. We recorded the packet generation time as well as the time when they are received at the sink node. Table I summarizes the simulation parameters set. MANER is compared with the following benchmark opportunistic protocols: Direct Delivery (DD), Spray and Wait (SnW), and ProPHET. We evaluate the performance of MANER algorithm against two metrics.

- Average latency: the difference between the time a data is sent at the source and time it is delivered at the destination (sink).

- Delivery ratio: the ratio of successfully delivered data to sink to total number of data transmitted by source nodes.

\section{B. Results and Analysis}

As far as wireless communication is concerned, animal physiological movement could be classified into two main states: (i) stationary state with no or relatively less mobility (e.g. grazing, graze-walking); and (ii) active state with high mobility (e.g. running from predators or illegal poachers) (Fig. 3.a). As shown in Figure 5, the mobility speed in each state are directly related to the link inter-contact time $(L)$ among nodes $[9,20]$. Shorter $L$ duration implies shorter contact time between nodes, which in-turn means a more frequent contact due to higher mobility speed [20]. Otherwise a longer $L$ implies the animals are moving slower (i.e. walking, sleeping, grazing) with less chance of contact to another node. Hence, RWP mobility depicts a faster and hectic movement 


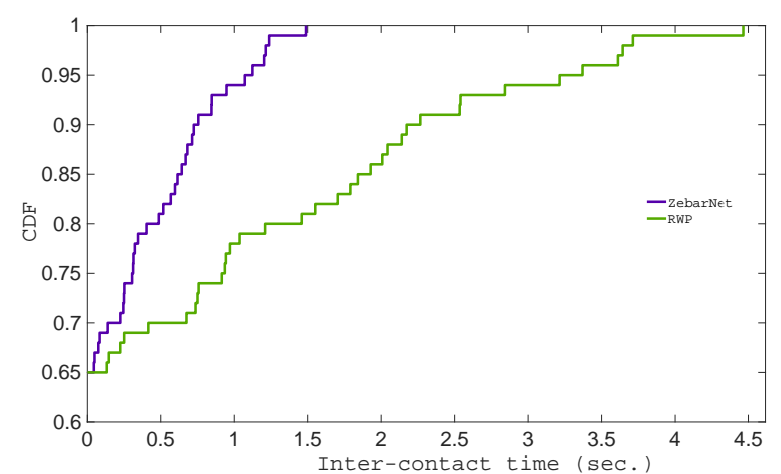

Fig. 5: Average inter-contact time $(L)$ distribution among nodes for ZebraNet and RWP mobility models.

model than ZebarNet (Fig. 5). In our case, MANER leverages $L$ to enable simple and reliable mesh networks among collared animals.

In monitoring applications events are relayed with high priority. In this aspect, one of the influential network parameters is time-to-live (TTL) and it highly impacts network delivery ratio and latency. However, it is not throughly studied in previous works [7]. Figure 4 shows that, an increase in TTL of a data improved the trend of delivery ratio in both $T^{+}{ }_{m g}$ cases for all the protocols. High TTL value is expected to increase the overall delivery ratio and vise versa [7].

In case of ZebraNet for higher TTL, MANER in particular, shows a relatively high delivery ratio compared to benchmark protocols. DD, PRoPHET and SnW show high delivery ratio with increasing TTL value, this is as expected, since for higher TTL, the chance of data reaching the intended destination would be higher. For both RWP and ZebraNET mobility pattern, the delivery ratio plot shows a pronounced change when the $T^{+}{ }_{m g}$ is high. This is mainly due to the inherent data replication properties of the routing protocols. MANER, SnW and PRoPHET are multi-copy, thus the number of duplicated data increases exponentially with higher TTL [7]. DD results in a lower delivery ratio due to the allowed one copy of data to be made, however, as can be seen from the latency plot, DD scores low latency (Fig. 6). The minimum observed delivery ratio for DD protocol is $20 \%$ and $45 \%$ respectively for RWP and ZebarNet mobility models at high $T^{+}{ }_{m g}$. MANER showed a maximum delivery ratio of approximately $90 \%$, this is because MANER leverages managed data replication to optimize the number of data duplications. Overall, packet delivery ratio of MANER is at least $12.3 \%$ (for ZebraNet) and $16.3 \%$ (for RWP) higher than of that of benchmark protocols. Furthermore, one can see that increasing packet generation rate leads to decreased difference between the delivery ratio performance of all protocols. In general, since data is replicated to all nodes in a network, the overall storage requirements for probabilistic routing becomes high, reducing the routing performance. Therefore, MANER routing is more suitable for wildlife opportunistic sensing.

Moreover, data average latency is lower for MANER, when compared to other protocols. Figure 6 shows the latency against TTL for both mobility models with $T^{+}{ }_{m g}$. Generally, the plot depicts that as the data TTL increases the overall message latency shows an increasing trend, this is in accordance with previous works [7]. Although DD and MANER have the lowest delivery ratio at high TTL, this is mainly because DD delivers data directly to the sink when it is in range than relaying it through neighboring nodes. However, MANER shows shortest average delay of $\approx 17 \mathrm{~s}$ and $\approx 1.5 \mathrm{~s}$ respectively for ZebarNet and RWP mobility models. Generally, SnW and PRoPHET have relatively higher average latency as shown in Fig. 6. This is due to the replication of data based on probability of contact, resulting in unnecessary flooding of data through out the entire network without optimization. ZebraNet model shows shorter average latency than RWP, supporting the hypothesis that opportunistic networks are applicable to wild life monitoring scenarios (Fig. 6). Overall, the average latency is decreased by up-to $65 \%$ when MANER is used.

\section{CONCLUSION}

In this paper, we proposed a dual radio IoT network architecture for wildlife monitoring. This approach facilitates ultra-low power IoT devices to be deployed for sustainable wildlife monitoring applications. In addition we presented a managed opportunistic protocol called MANER. In MANER, a node solely makes its decision for data forwarding based on local observations. In practice, it is near impossible, to determine a dissemination protocol for mobile network of wildlife monitoring systems. However, when subjected to diverse mobility patterns and traffic generation rate, MANER showed an improved performance than existing protocols. The evaluation results indicated that the proposed protocol outperforms the traditional algorithms. On average, our approach reduced the average latency by up-to $65 \%$. In addition the architecture improved network delivery ratio by up-to $16 \%$ for various packet traffic rates in the network. Therefore, for con-speciously sparse animal population, MANER is more optimal to deploy than utilizing existing opportunistic protocols. Moreover, in the future, we plan to validate these simulation results by performing a detailed implementation of the proposed scheme in a real world sensor devices by building a collar prototype with dual radio platform.

\section{ACKNOWLEDGMENT}

This research was supported by Smart Parks Project, funded by the Netherlands Organization for Scientific Research (NWO).

\section{REFERENCES}

[1] W. E. Cooper Jr. Escaping from predators: an integrative view of escape decisions. Cambridge University Press, 2015.

[2] J. K. Petersen. Understanding Technologies Surveillance Spy Devices, Their Origins \& Applications. CRC Press, 2002. 


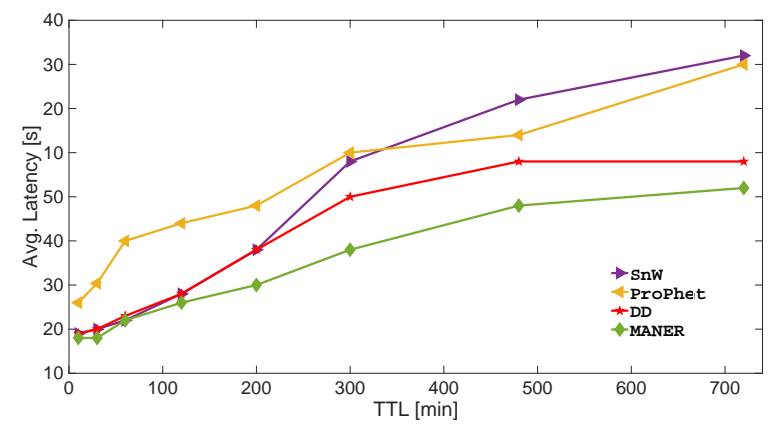

(a) RWP Avg. Lat. for High $T^{+}{ }_{m g}$

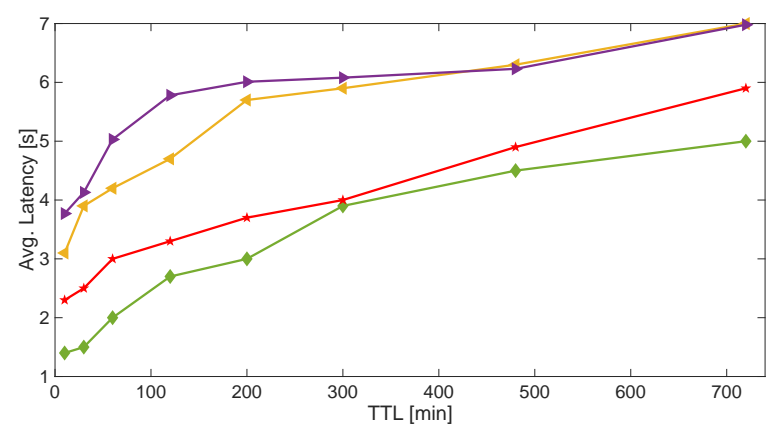

(c) ZebraNet Avg. Lat. for High $T^{+}{ }_{m g}$

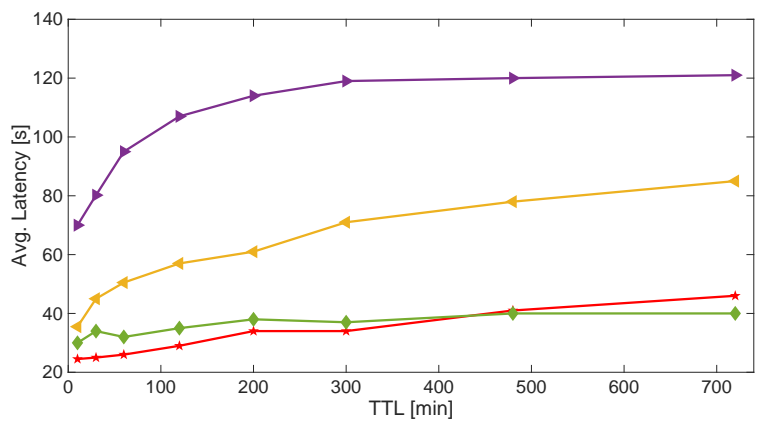

(b) RWP Avg. Lat. for Low $T^{+}{ }_{m g}$

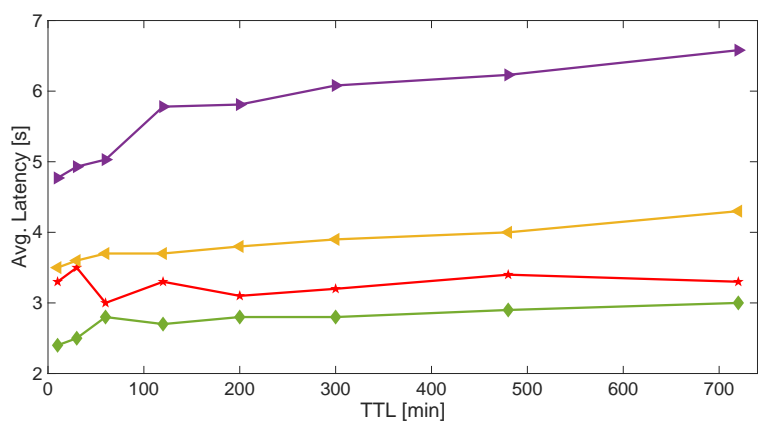

(d) ZebraNet Avg. Lat. for Low $T^{+}{ }_{m g}$

Fig. 6: Average Latency for RWP and ZebraNet mobility models

[3] P. O'Donoghue and C. Rutz. Real-time anti-poaching tags could help prevent imminent species extinctions. Journal of Applied Ecology, 53(1):5-10, 2016.

[4] J. Polastre, R. Szewczyk, A. Mainwaring, D. Culler, and J. Anderson. Analysis of wsn for habitat monitoring. In WSNs, pages 399-423. Springer, 2004.

[5] C. Jones, B. Warburton, J. Carver, and D. Carver. Potential applications of wsns for wildlife monitoring programs. Wildlife Society Bulletin, 39(2):341-348, 2015.

[6] S. Wu and Y. Tseng. Wireless ad hoc networking: personal-area, local-area, and the sensory-area networks. CRC Press, 2007.

[7] Payal J. and Rachna S. A survey on opportunistic routing protocols for wireless sensor networks. Procedia Computer Science, 79:603 - 609. ICCCV 2016.

[8] S. Pathak, N. Gondaliya, and N. Raja. A survey on prophet based routing protocol in dtn. In ICEI, pages 110-115, Feb 2017.

[9] P. Juang, H. Oki, Y. Wang, M. Martonosi, L. Peh, and D. Rubenstein. Zebranet. ACM SIGARCH, 30(5):96-107, 2002.

[10] O. Landsiedel, J gila B., K Wehrle, J. Thiele, and H. Mallot. Rat watch. ACM REALWSN, 2006.

[11] A. Al-Hinai, H. Zhang, Y. Chen, and Y. Li. Tb-snw. The Journal of Supercomputing, 69(2):593-609, Aug 2014.

[12] A. Keränen, J. Ott, and T. Kärkkäinen. The one simulator for dtn protocol evaluation. In IC, ICST, 2009.

[13] N. Sornin, M. Luis, T. Eirich, T. Kramp, and O. Hersent. Lorawan specifications. LoRa Alliance, 2015.

[14] S. Raza, P. Misra, Z. He, and T. Voigt. Building the internet of things with bluetooth smart. Elsevier, Ad Hoc Networks, 57:19-31, 2017.

[15] E. D. Ayele, C. Hakkenberg, J. P. Meijers, K. Zhang, N. Meratnia, and P. J. M. Havinga. Performance analysis of lora radio for an indoor iot applications. In $I o T G C$, pages 1-8, July 2017.

[16] P. Kindt and S. Chakraborty. Neighbor discovery latency in ble-like duty-cycled protocols. CoRR, abs/1509.04366, 2015.

[17] B. K. Saha, S. Misra, and S. Pal. Seer. TMC, 16(10):2876-2888, Oct 2017.

[18] E. D. Ayele, N. Meratnia, and P. J. M. Havinga. Hama: A herd-movement adaptive mac protocol for wireless sensor networks. In NTMS, pages 1-7, Nov 2016.

[19] R. Jovani and R. Mavor. Group size versus individual group size frequency distributions. Animal Behaviour, Elsevier, 82(5):1027 - 1036, 2011.

[20] O. Sistla, A.and Wolfson and B. Xu. Opportunistic Data Dissemination in Mobile P2P Networks, pages 346-363. Springer Berlin Heidelberg, Berlin, 2005. 